\title{
Reflexiones sobre el EEES tras los primeros cursos de la asignatura Arquitectura de Computadores
}

\author{
Julio Ortega, Mancia Anguita, Jesús González, Miguel Damas \\ Departamento de Arquitectura y Tecnología de Computadores \\ E.T.S.I.I.T., Universidad de Granada \\ \{jortega, manguita, jesusgonzalez, mdamas\}@ugr.es
}

\begin{abstract}
Resumen. Han pasado ya algunos cursos académicos desde que empezaron a impartirse en la Universidad de Granada estudios de grado según las directrices del Espacio Europeo de Educación Superior (EEES). En el caso del grado en Ingeniería Informática, este curso 2015/2016 terminará la tercera promoción. En este artículo, tras resumir los objetivos de la denominada reforma de Bolonia, se describen algunas de las situaciones en las que se ha traducido la implementación de la reforma en nuestro país. Para extraer algunas de las conclusiones del artículo se ha tomado como referencia la asignatura de Arquitectura de Computadores de segundo del grado en Ingeniería Informática que está siendo impartida por varios de los autores del artículo.
\end{abstract}

Palabras clave: Arquitectura de Computadores, Especio Europeo de Educación Superior (EEES), Evaluación del aprendizaje, Mapas conceptuales.

\begin{abstract}
It has been several academic years since the beginning of study programmes at the University of Granada according to the guidelines of the European Higher Education Area. In this paper, after summarizing the main objectives of the so-called Bologna reform, some of the consequences of its implementation in Spain are described. Moreover, the subject of Computer Architecture, taught in the second course by some of the authors of the paper, is considered as a reference to extract some of the conclusions of the paper.
\end{abstract}

Keywords: Computer architecture, Conceptual maps, European Higher, Eduaction Area, Learning assessment.

\section{Introducción}

En este curso 2015/2016 se graduará la tercera promoción del Grado en Ingeniería Informática y la primera del Máster en Ingeniería Informática de la Universidad de Granada, ambas impartidas en la Escuela Técnica Superior de Ingenierías Informática y Telecomunicaciones (ETSIIT). Cabría preguntarse si estos nuevos planes de estudio en Ingeniería Informática, regulados bajo las directrices del Espacio Europeo de Educación Superior (E.E.E.S.), han contribuido a mejorar las calidad de la docencia 
impartida y, como resultado, la formación de los titulados en Informática. Sin duda, proporcionar una respuesta científicamente concluyente a esta cuestión necesita un análisis amplio tanto de los resultados de la evaluación de los estudiantes, como del éxito con que las competencias adquiridas están dando respuesta a las demandas sociales propias de cada grado. Si bien un estudio de este calibre exige bastante más información de la que se dispone actualmente, es posible analizar ya algunos de los datos obtenidos a partir de la docencia en las asignaturas que se han impartido ya durante algunos cursos, y poner de manifiesto alguno de los efectos de las nuevas estrategias docentes que se están poniendo en práctica. En esa línea, este trabajo utiliza algunos de los resultados disponibles de la asignatura Arquitectura de Computadores del Grado en Informática, para extraer, tras los cuatro años en los que se ha impartido, algunas conclusiones respecto a la implantación de los principios del EEES y sus resultados en el aprendizaje de los contenidos de la asignatura.

Así, en la Sección 2 se revisarán los principios y los objetivos que se pretendían alcanzar con la implantación del EEES, y se confrontan con algunas de las conclusiones respecto al aprendizaje que se están extrayendo de estudios recientes en neurociencia. A continuación, la Sección 3 describe los contenidos y la metodología con la que se imparte la asignatura obligatoria Arquitectura de Computadores del segundo cuatrimestre del segundo curso del grado en Ingeniería Informática, para analizar en la Sección 4 los resultados de evaluación de los cursos en los que se viene impartiendo, en algunos de los grupos de la asignatura. Finalmente, la Sección 5 proporciona las conclusiones del trabajo y la Sección 6 contiene las referencias bibliográficas.

\section{Qué es y qué ha supuesto la implantación del EEES}

En [1] se hace una presentación bastante acertada de los objetivos del EEES, conocido usualmente como la reforma o proceso de Bolonia. En ese trabajo se hace hincapié fundamentalmente en las consecuencias sobre la metodología docente del nuevo marco que, en la UGR, se empezó a poner en práctica en el curso 2010/2011, y más concretamente sobre la enseñanza/aprendizaje de las asignaturas de Arquitectura y Tecnología de Computadores.

En realidad, el EEES se plantea como un proceso, iniciado en 1999, para armonizar los distintos sistemas universitarios de la Unión Europea, de forma que se facilitase la movilidad de los estudiantes en el marco de la globalización socio-económica desde el que se contemplaba el siglo XXI, antes de la crisis de 2008. Las reformas que se requerían en muchos países para ajustar sus sistemas a lo establecido en la Declaración de Bolonia se contempló como una oportunidad para implementar cambios en las metodologías docentes que han afectado no solo a la estructura curricular y a la movilidad de los estudiantes, sino también al proceso de enseñanza/aprendizaje y a organización del personal docente, entre otros factores. El establecimiento del sistema de créditos ECTS (European Credit Transfer System) en el que se tiene en cuenta la carga de trabajo del estudiante fuera de las clases 
presenciales ha supuesto un cambio importante a la hora de organizar los contenidos desde una especie de contrato entre profesor y estudiante, que contribuye a la transparencia de un sistema centrado en el aprendizaje del estudiante. Así, en nuestro país, el EEES se ha contemplado no solo como un cambio en los planes de estudios sino también como una oportunidad para mejorar la calidad de la Universidad española.

En la práctica la implantación EEES ha tenido unos efectos muy concretos desde el punto de vista de la organización de las asignaturas. Así, se deben especificar las competencias que debe alcanzar el estudiante a través de la asignatura en cuestión, y elaborar guías docentes como instrumento para la transparencia, y como marco de referencia para profesores y estudiantes del proceso de enseñanza-aprendizaje. Además, el interés por las metodologías docentes ha generado un número considerable de publicaciones docentes y la financiación de proyectos de innovación docente para concretar el enfoque de aprendizaje basado en el estudiante.

Otros cambios relacionados de una u otra forma con la reforma de Bolonia incluyen la puesta en marcha de Agencias de Calidad, Vicerrectorados de Calidad, y Comisiones de Calidad de los Títulos. Uno de los resultados de la búsqueda de transparencia en los procesos Universitarios ha sido la elaboración de memorias de Verificación de los Títulos. Éstas recogen no solamente las asignaturas sino también datos de infraestructuras, profesorado que los imparte, y previsiones en cuanto a los indicadores de calidad y rendimiento relacionadas con las tasas de titulados que terminan respecto a los matriculados, etc. Así, para que pueda impartirse un título universitario se debe obtener la correspondiente acreditación sometiendo su memoria de verificación a la evaluación de la agencia nacional o de la comunidad autónoma correspondiente y, una vez aprobado y tras impartirse durante unos cursos debe nuevamente volverse a evaluar para obtener la renovación de la acreditación. En estos últimos años, también se ha dedicado una atención considerable a los procesos de evaluación del profesorado. Además de la implantación de procesos de acreditación para poder acceder a las distintas figuras docentes, las Universidades han puesto en marcha programas de evaluación de la calidad docente que incluyen encuestas de satisfacción de los estudiantes. Una de las consecuencias de las nuevas iniciativas que se han implantado es el incremento del trabajo de gestión asociado a la necesidad de generar y evaluar los documentos necesarios que aseguren la transparencia en la enseñanza Universitaria.

Como se ha dicho antes, la reforma de Bolonia también aborda cuestiones relacionadas con las metodologías docentes. De hecho, se promueve que el profesor aplique la metodología más adecuada para los contenidos de la asignatura teniendo en cuenta la relevancia que se da ahora al papel del estudiante como protagonista de su propio aprendizaje. En general, se favorece el aprendizaje basado en problemas a través del que el propio estudiante construye sus mapas de relaciones entre los conceptos de la asignatura, a partir de los elementos fundamentales presentados por el profesor, que se contempla ahora como orientador y evaluador del proceso de aprendizaje. Se pretende reducir el protagonismo de la lección magistral, que se suele identificar con el aprendizaje memorístico por parte del estudiante, pero que tiene 
efectos bastante positivos si se introducen elementos que promuevan su participación activa en el desarrollo de las clases y la resolución de ejercicios y problemas con los que se va ilustrando la utilidad de los conceptos explicados. Desde nuestro punto de vista, los problemas de las clases magistrales provienen de la masificación de los grupos y de que no se ponga de manifiesto, a través de esas clases, la relación entre los conocimientos teóricos y los problemas que pueden abordarse gracias a ellos.

Uno de los resultados de las discusiones sobre dichas metodologías ha sido la instauración generalizada de la evaluación continua. La necesidad de realizar actividades de evaluación continua surge al cuestionar que mediante un examen final sea posible medir los resultados de aprendizaje que se establecen en las guías, y aun en caso de que puedan evaluarse, por la necesidad de plantear actividades que preparen al estudiante para alcanzar dichos resultados. Si se busca que los estudiantes sean capaces de resolver determinado tipo de problemas, es conveniente que se les planteen a través de relaciones de problemas y se discuta en clase la forma de abordarlos. En la mayoría de las asignaturas de las carreras científicas o técnicas ya se acostumbraba a acompañar cada tema con una relación de problemas, y se dedicaban clases a resolverlos. Además, normalmente ya se organizaba la docencia entre clases de teoría y prácticas, y en estas últimas los estudiantes se enfrentaban a problemas experimentales cuya mayor o menor aplicabilidad a casos reales estaba más bien limitada por la falta de recursos que por razones metodológicas.

Otra razón importante para que se realicen pruebas de evaluación a lo largo del curso es que los estudiantes reciban realimentación acerca de si lo que han realizado es correcto o incorrecto: la denominada evaluación formativa. En cualquier caso, la evaluación continua no significa que haya que evaluar a los estudiantes a través de pruebas parciales. De lo que se habla, más bien, es de la incorporación en la nota final de las calificaciones de las pruebas realizadas a lo largo del curso, siempre y cuando el estudiante alcance un mínimo en las pruebas presenciales, que se aconseja que sea una calificación próxima a la necesaria para aprobar la asignatura [2].

Sin embargo, la coincidencia de la impartición de un número considerable de asignaturas que implementan evaluación continua junto con la disponibilidad de apuntes, transparencias y demás material pueden afectar negativamente al aprendizaje, como veremos a continuación.

\subsection{Una perspectiva del EEES desde la neurociencia}

El aprovechamiento de las tecnologías de la información y las comunicaciones ha sido un elemento esencial para mejorar la calidad de la enseñanza universitaria. Plataformas docentes de fácil acceso y uso como SWAD [3] han contribuido a difundir y acceder a material docente, mejorar la comunicación profesor-estudiante, y facilitar la gestión de los grupos, entre otras facetas de la actividad docente. Por otra parte Internet y el acceso a revistas electrónicas posibilitan la actualización de material y disponer de herramientas de acceso abierto. Actualmente, es usual que los estudiantes dispongan de las transparencias de clase y soluciones a problemas propuestos. Han quedado atrás los años en que era fundamental asistir a clase para 
tomar apuntes que recogiesen los contenidos de la asignatura y las explicaciones del profesor. Si se faltaba a alguna clase había que recurrir a las notas prestadas por algún compañero o a extraer la información de la bibliografía recomendada. Como se dispone de las transparencias y de gran cantidad de información muchos estudiantes no se preocupan de tomar apuntes y consideran que, en su momento, podrán asimilarla. Como si se tratase de copiar un fichero desde un directorio (en el disco) a otro (en el cerebro).

Sin embargo la neurociencia nos dice que nuestra memoria no funciona como el disco duro de nuestros computadores. De hecho, los modelos más aceptados actualmente indican que el aprendizaje se construye, y la memorización es el primer paso de un proceso de síntesis personal de lo que se ha leído, escuchado, visto, etc., que implica creatividad y juicio [4]. Los eventos que se introducen en la memoria a corto plazo se transforman en pensamientos memorizados y pasan a la memoria a largo plazo una vez transcurrido un cierto tiempo [5]. Estos dos tipos de memoria se basan en procesos biológicos diferentes y se ha puesto de manifiesto que el paso a la memoria a largo plazo no sólo cambia la concentración de neurotransmisores para reforzar ciertas conexiones, sino que también aparecen nuevas sinapsis, implicando cambios anatómicos además de bioquímicos. Por tanto, debe activarse un gen para producir dichas proteínas y responder al proceso de aprendizaje. Además, la consolidación de la memoria a largo plazo implica una cadena de interacciones relativamente larga entre el hipocampo y el córtex cerebral. La atención está en la base de la consolidación de la memoria al ocasionar que las neuronas del córtex envíen señales y se abra un camino para que la dopamina llegue al hipocampo y permita la consolidación de la memoria explícita al activar los genes que estimulan la síntesis de nuevas proteínas.

Se debería promover el estudio interrelacionado de los contenidos de las asignaturas junto con los de otras asignaturas, y plantear preguntas, prácticas y problemas que muestren la relación entre los distintos conceptos y faciliten indexar la información que tenemos en la memoria. En esta línea, sería conveniente fomentar el aprendizaje interrelacionado de distintas asignaturas. Sin embargo, la actual implementación de los Planes de Estudio de los grados se basa en asignaturas impartidas por profesores encuadrados en Departamentos. Cada Departamento es el responsable de organizar la docencia de las asignaturas de un mismo ámbito de conocimiento en los distintos estudios. Si bien los Planes de estudio ordenan en el tiempo la impartición de las distintas asignaturas en función de las dependencias entre sus contenidos, usualmente no se pone de manifiesto la interrelación que existe entre contenidos de asignaturas que se imparten simultáneamente. Es más, el número de exámenes y de actividades a realizar puede ser tan elevado, que es difícil que el estudiante profundice en los conceptos y pueda asentar lo aprendido, y se produce una fuerte competencia entre el tiempo que los estudiantes dedican a cada asignatura. Si en una asignatura se realizan exámenes parciales que permiten a los estudiantes que los superan no tener que volverse a evaluar de los contenidos del examen parcial en una prueba que abarca toda la asignatura, las demás asignaturas deberían tener en cuenta esta circunstancia dado que, como se viene observando, los estudiantes suelen concentrarse en aprobar los exámenes parciales a los que se les convoca, y les resulta más difícil mantener el 
ritmo de las demás asignaturas. Si además, pueden disponer del material de la asignatura a través de una plataforma docente, la reacción usual será la de pensar que pueden preparar los exámenes, sin tener que seguir muy exhaustivamente las explicaciones de clase.

\section{Arquitectura de Computadores (AC) en la UGR tras el EEES}

La asignatura de Arquitectura de Computadores se imparte en el segundo curso del grado en Ingeniería Informática de la Universidad de Granada. Se trata de una asignatura obligatoria cuyos objetivos y contenidos se han descrito en [6,7]. La guía docente de la asignatura se puede encontrar en la dirección [8].

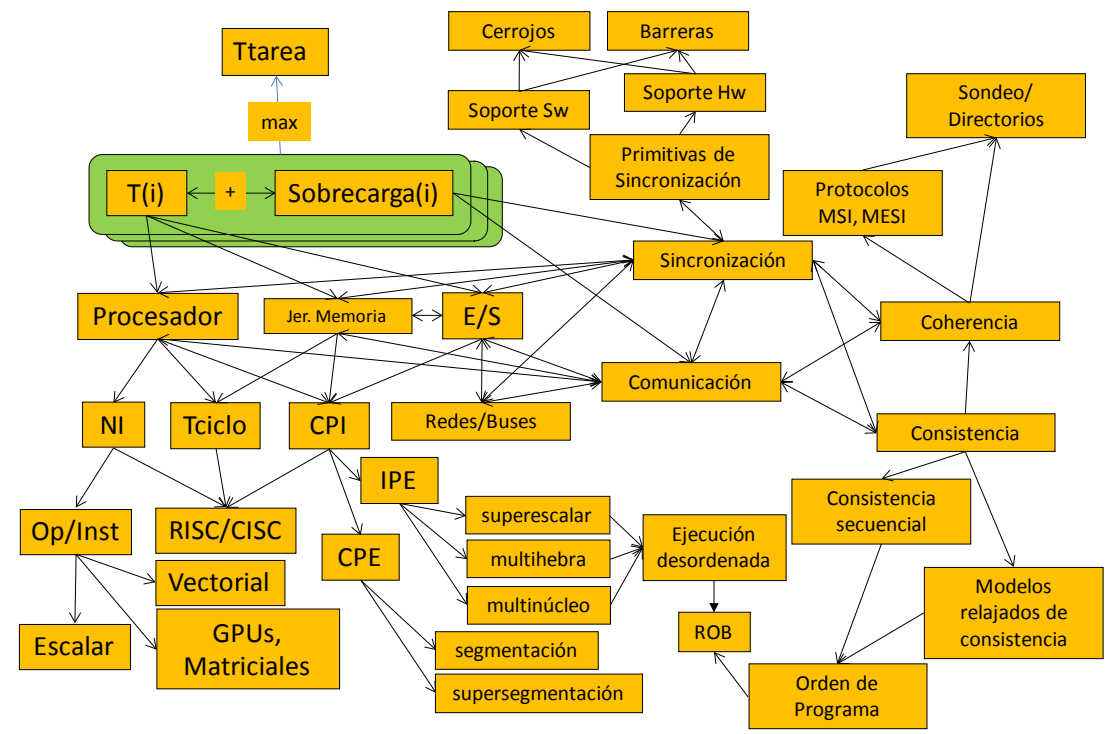

Figura 1. Un mapa conceptual para la asignatura Arquitectura de Computadores del Grado en Ingeniería Informática de la UGR [9]

Los objetivos de la asignatura proponen el estudio de la arquitectura del computador desde el punto de vista del aprovechamiento del paralelismo que implementan los computadores actuales, más que desde el punto de vista del diseño. A la vez que se introducen los conceptos fundamentales de las arquitecturas paralelas que necesita un ingeniero de computadores, esta perspectiva muestra la importancia de conocer las características de la arquitectura del computador para desarrollar aplicaciones eficientes, y justifica una asignatura obligatoria de este tipo en el segundo curso del grado en Ingeniería Informática. La Figura 1 muestra un marco conceptual de la asignatura, cuyos contenidos se estructuran en cinco temas dedicados, respectivamente a la evaluación de las prestaciones, el procesamiento paralelo, las arquitecturas con paralelismo a nivel de hebra, las arquitecturas con paralelismo entre 
instrucciones, y una introducción a las arquitecturas actuales con paralelismo de datos.

La asignatura se imparte a través de grupos de teoría, cada uno de los cuales tiene asignados varios grupos de prácticas de alrededor de 25 estudiantes. La evaluación de la asignatura incluye una calificación de hasta 6 puntos de la parte de teoría y otra de hasta 4 puntos de la parte de prácticas. Para sumar las dos calificaciones es necesario que superen un $40 \%$ de la nota máxima de cada apartado (2.4 en la parte de teoría y problemas, y 1.6 en la parte de prácticas) y para aprobar la asignatura es necesario obtener una calificación total de 5.0 o más puntos. La evaluación de la parte de teoría se realiza a través de una prueba escrita de todos los contenidos de la asignatura, a la que se asigna una calificación máxima de 4 puntos, y de varias pruebas parciales (cuatro pruebas de la teoría de los diferentes temas y una prueba parcial de problemas) en las que se puede alcanzar una calificación máxima de 2.0 puntos. Las prácticas se evalúan mediante una prueba escrita de todos los contenidos, que tiene una calificación máxima asignada de 2.0 puntos, y entregas y evaluaciones de las prácticas en el aula, con una calificación máxima de 2 puntos.

Tabla 1. Descripción de los grupos analizados

\begin{tabular}{|l|c|l|c|}
\hline Código & Grupo & Estudios (Curso Académico) & Estudiantes \\
\hline 12A & $\mathrm{A}$ & \multirow{3}{*}{ Ing. Informática (2011-12) } & 53 \\
\hline 12B & $\mathrm{B}$ & & 31 \\
\hline 12C & $\mathrm{C}$ & & 24 \\
\hline 13A & $\mathrm{A}$ & \multirow{2}{*}{ Ing. Informática (2012-13) } & 73 \\
\hline 13B & $\mathrm{B}$ & & 65 \\
\hline 13IM & & \multirow{2}{*}{ Ing. Informática y Matemáticas (2012-13) } & 23 \\
\hline 14A & $\mathrm{A}$ & \multirow{2}{*}{ Ing. Informática (2013-14) } & 73 \\
\hline 14B & $\mathrm{B}$ & & 72 \\
\hline 14IM & & Ing. Informática y Matemáticas (2013-14) & 25 \\
\hline 15A & $\mathrm{A}$ & \multirow{2}{*}{ Ing. Informática (2014-15) } & 88 \\
\hline 15B & $\mathrm{B}$ & & 99 \\
\hline 15IM & & Ing. Informática y Matemáticas (2014-15) & 30 \\
\hline
\end{tabular}

\section{Resultados experimentales de la docencia en AC}

En esta sección se proporcionan algunos resultados de los rendimientos de los estudiantes en las distintas pruebas de teoría de la asignatura. Corresponden a los cuatro cursos académicos en los que la asignatura se ha impartido (2011/2012 a 2014/2015) y a varios grupos de teoría impartidos por uno de los autores del artículo. En la Tabla 1 se proporciona el número de estudiantes de los grupos considerados, los estudios a los que pertenecen y el curso académico al que corresponden. La evaluación en los cursos 2013-2014 y 2014-2015 se han realizado según el procedimiento de evaluación continua detallado en la guía de la asignatura [8] y descrito en la Sección 3. La Figura 2 muestra las medias de las calificaciones de teoría (examen en los cursos 2011-12 y 2012-13 y examen más pruebas de clase en los 
cursos 2013-14 y 2014-15) de distintos grupos. Como se puede observar, existen cambios en los valores medios, pero no se observa una tendencia uniforme a partir de que se implanta la evaluación continua. A continuación se comprueba si las diferencias son estadísticamente significativas.

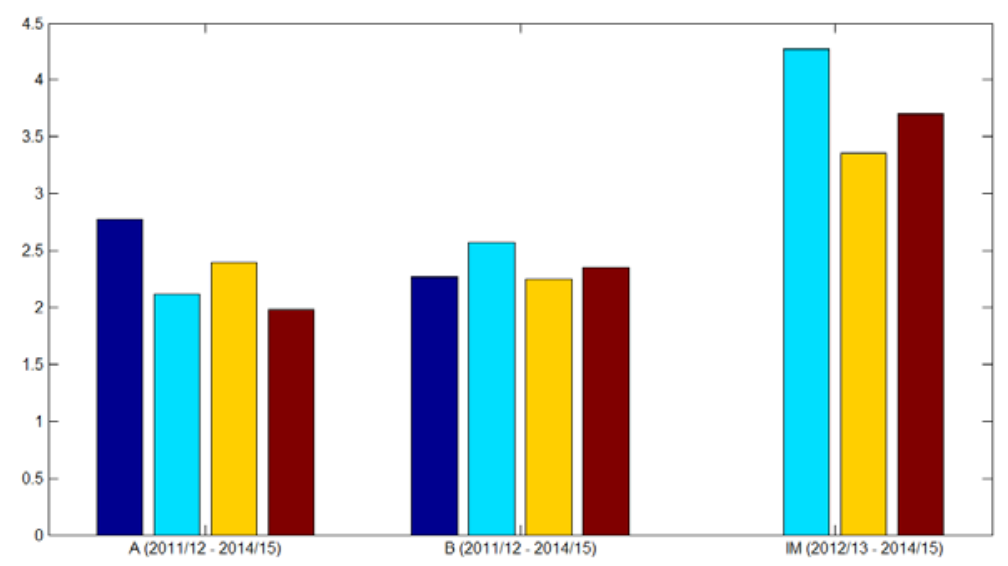

Figura 2. Medias de las calificaciones de teoría (entre 0 y 6 ) en los distintos cursos académicos para los distintos grupos: A y B del grado en Ingeniería Informática y grupo del grado en Ingeniería Informática y Matemáticas (IM)

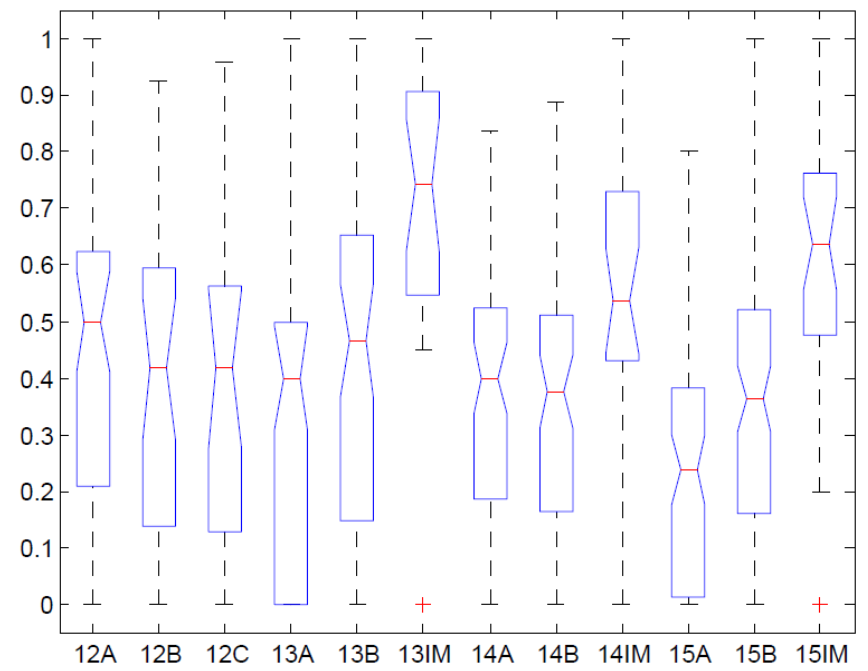

Figura 3. Medias y desviaciones de las calificaciones de teoría para cada uno de los cursos (respecto a la calificación máxima del examen) 


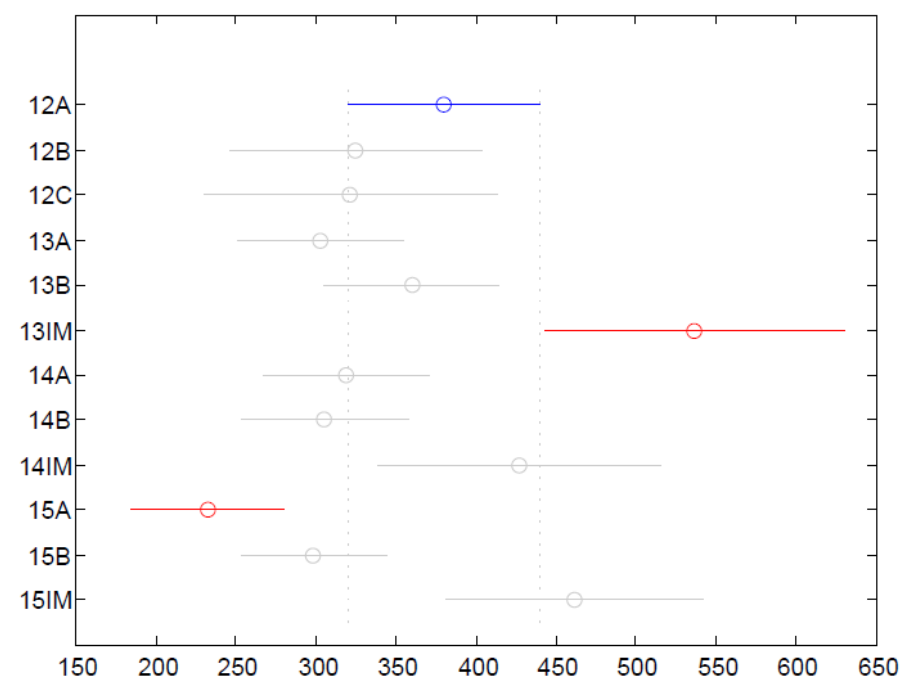

Figura 4. Análisis estadístico de la significación de las diferencias en la Figura 2 mediante el test de Kruskal-Wallis

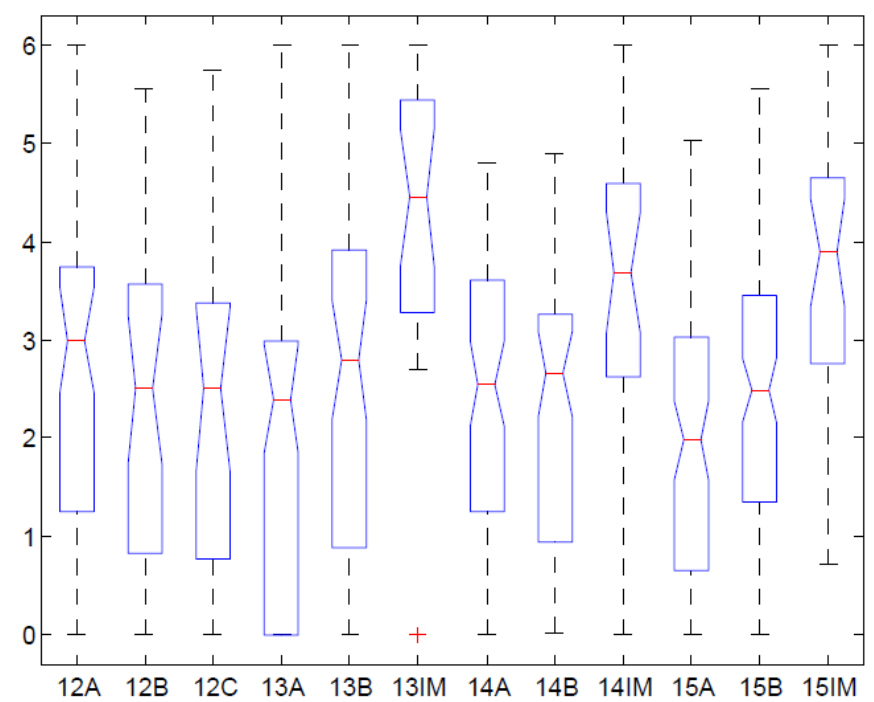

Figura 5. Medias y desviaciones de las calificaciones totales de teoría para cada uno de los cursos 


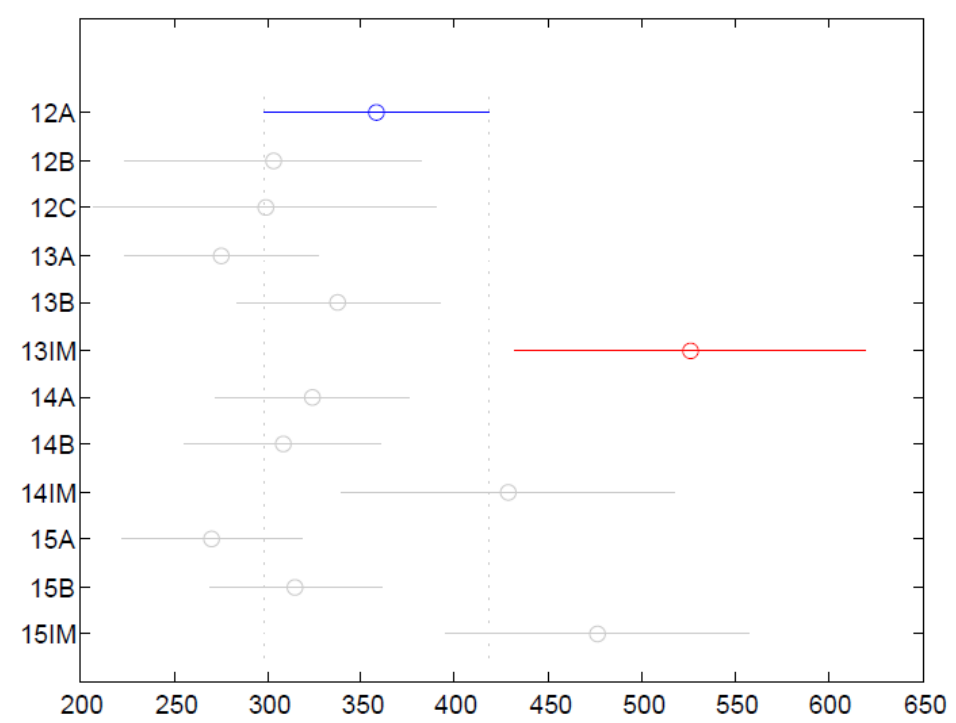

Figura 6. Análisis estadístico de la significación de las diferencias en la Figura 4 mediante el test de Kruskal-Wallis

Los resultados de las Figuras 3 y 4 comparan las fracciones de las notas máximas del examen de teoría y problemas que abarca todos los contenidos de la asignatura. En los cursos académicos 2011-2012 y 2012-2013 este examen tenía asignada una calificación máxima de seis puntos, mientras que desde la implantación de la evaluación continua la nota máxima era de 4 puntos. En la Figura 3 se muestran las medias y las desviaciones a través de los distintos grupos y años. Si se comparan por un lado los grupos del grado en ingeniería informática, y por otro los grupos del grado en ingeniería informática y matemáticas, se puede apreciar una ligera disminución en los valores medios de las calificaciones del examen de toda la asignatura en los cursos en los que se ha utilizado la evaluación continua. Se podría pensar en algún tipo de despreocupación por el estudio del examen que abarca toda la asignatura por tener algunas notas de clase de partida. Sin embargo, el análisis estadístico que se ha realizado aplicando un test de Kolmogorov-Smirnov, nos ha permitido comprobar que los datos no siguen una distribución normal en ninguno de los grupos y cursos, y el posterior test de Kruskal-Wallis nos indica (Figura 4) que las diferencias solo son estadísticamente significativas para algunos grupos en algún curso académico y no están asociadas al cambio a un sistema de evaluación continua en el curso 2013-2014.

En las Figuras 5 y 6 se comparan los resultados del examen de teoría de seis puntos en los cursos 2011-2012 y 2012-2013 con la calificación de teoría total obtenida al sumar el examen de cuatro puntos y los puntos (hasta un máximo de dos) correspondientes a las pruebas de evaluación continua. En este caso la situación sigue siendo muy similar a la que se refleja cuando se comparan los porcentajes de calificaciones de los exámenes. Existen diferencias entre los valores medios obtenidos por los distintos grupos pero no son diferencias estadísticamente significativas que se puedan asociar al cambio en el sistema de evaluación. Las diferencias significativas se producen entre 
el grupo del grado en ingeniería informática y matemáticas del curso académico 2012-2013 y los demás. En los grupos de este grado suelen observarse calificaciones medias más elevadas que en los grupos del grado en ingeniería en informática, aunque también hay que tener en cuenta que existen diferencias apreciables entre las notas medias de acceso a cada una de estas titulaciones.
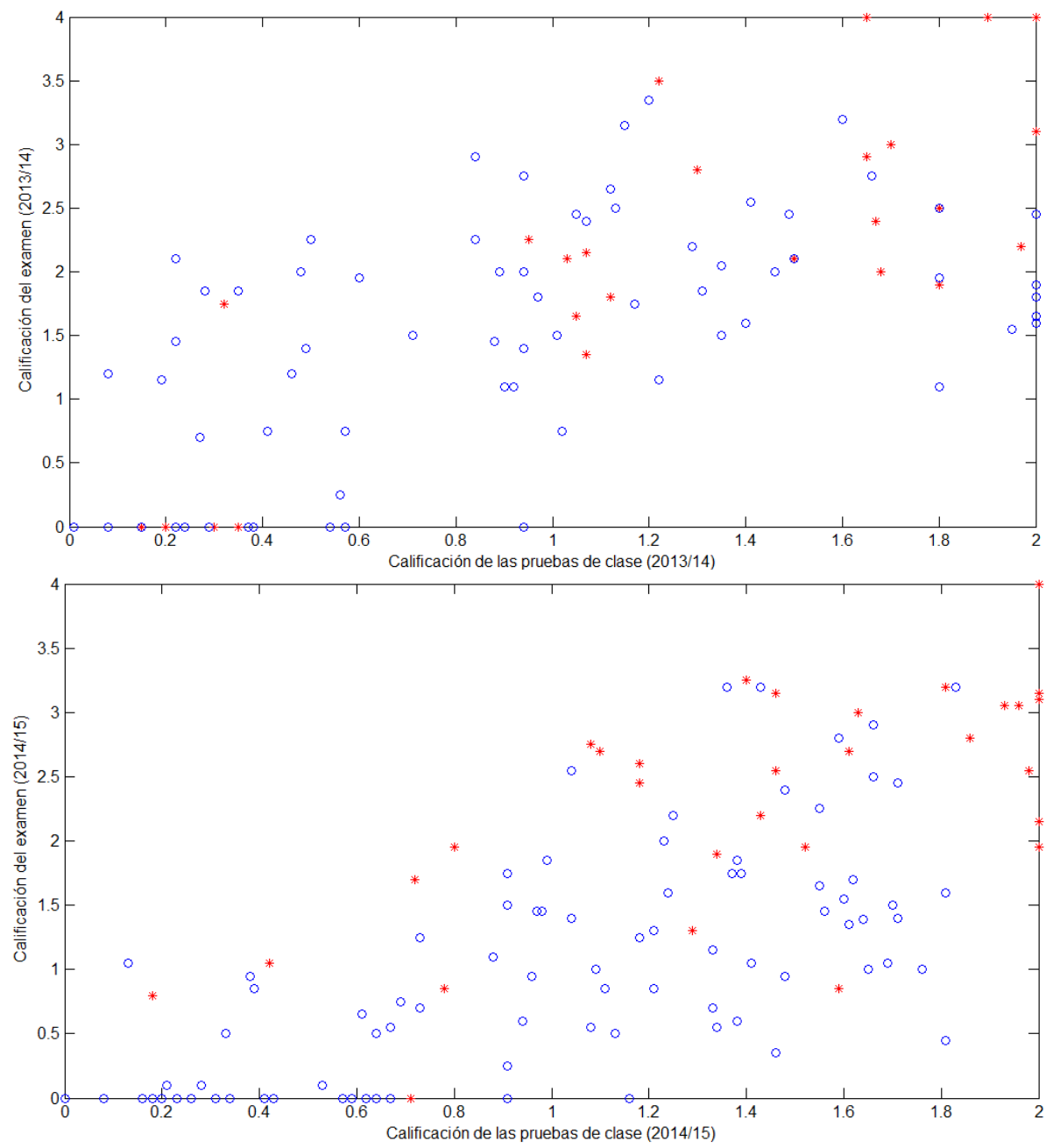

Figura 7. Relación entre calificaciones de clase y del examen de los estudiantes para el grupo A del grado en Ingeniería Informática ('o’) y del grado en Ingeniería Informática y Matemáticas ('*’)

En la Figura 7, cada punto corresponde a las calificaciones de las pruebas de clase y la del examen de teoría de un estudiante. Se puede ver que en general, calificaciones más elevadas en las pruebas de clase suelen coincidir con calificaciones mayores en los exámenes de toda la materia. Además, se ha comprobado que existe una 
correlación significativa entre dichas calificaciones en todos los grupos y para los dos cursos académicos en los que se ha aplicado la metodología de evaluación continua: coeficientes correlación de 0.696, 0.693, y 0.669 en el curso 2013/2014 y 0.586, 0.528 , y 0.808 en el curso 2014/2015, respectivamente para los grupos A y B del grado en Informática y el grupo del grado en Ingeniería Informática y Matemáticas).

\section{Conclusiones y propuestas}

Los datos de las calificaciones obtenidas por los estudiantes en la asignatura Arquitectura de Computadores desde que empezó a impartirse en el curso 2011/2012 ponen de manifiesto que no existen diferencias significativas entre las calificaciones de teoría obtenidas tras la aplicación de la evaluación continua en el curso 2013/2014. Tampoco parecen afectar a las calificaciones que los estudiantes obtienen en el examen de teoría y, además, la correlación observada entre las calificaciones de las pruebas de clase y las calificaciones del examen aconsejan la realización de estas pruebas que resultan útiles desde el punto de vista de la evaluación formativa del estudiante.

Además, tras las conclusiones acerca de las características del proceso de aprendizaje a las que se ha llegado tras los últimos avances de la neurociencia, se puede concluir que, es necesario el trabajo continuado sobre los conceptos que aborda una asignatura, pero también es importante que existan pruebas globales en las que se evalúen todos los contenidos, dada la necesidad de promover que los estudiantes creen mapas conceptuales que afiancen la asimilación de los conceptos de la asignatura y su ubicación en la memoria. Puede ser conveniente que los resultados de las pruebas que se realizan a los estudiantes se tengan en cuenta en la calificación final, pero debe prestarse la suficiente atención a las perturbaciones que se pueden originar en la marcha de otras asignaturas si la parte de la calificación que se asigna a esas pruebas es demasiado elevada.

En general, se puede afirmar que salvo la mayor transparencia asociada a la elaboración de las guías de las asignaturas y los procesos de verificación de los estudios, el EEES no ha supuesto cambios considerables en cuanto a la forma que se impartían las asignaturas en los estudios de ciencias e ingenierías donde ya se dedicaba un tiempo considerable a las clases prácticas y a la realización de problemas. La esperada mejora, relacionada con una reducción del tamaño de los grupos que permitiría una atención más personalizada del estudiante, no ha llegado. Quizá la coincidencia de la puesta en marcha de la reforma con las consecuencias de la crisis económica de 2008 ha tenido bastante que ver.

\section{Referencias}

1. Diaz, J; Morillas, C.; Romero, S.; Guillén, A.:”Bolonia for dummies”. Enseñanza y Aprendizaje de Ingeniería de Computadores, No.1, pp.5-17, 2011. 
2. UNED (Vicerrectorado de Espacio Europeo y Planificación Docente y Vicerrectorado de Calidad e Innovación Docente): “Evaluación Continua en el EEES”

http://portal.uned.es/pls/portal/docs/PAGE/UNED_MAIN/LAUNIVERSIDAD/VICERRECTORAD OS/CALIDAD E INTERNACIONALIZACION/INNOVACION DOCENTE/IUED/DOCUMENT OS/EVALUACION_CONTINUA_EEES_UNED.PDF

3. SWAD (Sistema Web de Apoyo a la Docencia), swad.ugr.es, 2016.

4. Bain, K.:"Lo que hacen los mejores profesores universitarios". Publicacions Universitat de València, 2006.

5. Kandel, E.R.:"En busca de la memoria: una nueva ciencia de la mente". Katz Editores, 2007.

6. Anguita, M.; Cañas, A.; Fernández, F.J.; Ortega, J.; Rojas, I..”El perfil de Ingeniería de Computadores y las asignaturas de Estructura y Arquitectura de Computadores en el grado de Ingeniería Informática”. Enseñanza y Aprendizaje de Ingeniería de Computadores, №.1, pp.89-98, 2011.

7. Ortega, J.; Anguita, M.:”Arquitectura de Computadores en seis créditos ECTS”. Enseñanza y Aprendizaje de Ingeniería de Computadores, No.2, pp.13-25, 2012.

8. Guía docente de Arquitectura de Computadores (grado en Ingeniería Informática de la UGR). http://grados.ugr.es/informatica/pages/infoacademica/guias_docentes/201516/segundo/2semestre/arqu itecturadecomputadores/! 2016.

9. Ortega, J.; Anguita, M.:"Relatos, Mapas Conceptuales y Arquitectura de Computadores”. Enseñanza y Aprendizaje de Ingeniería de Computadores, Nº.4, pp.89-102, 2014. 